EPJ Web of Conferences 106, 05006 (2016)

DOI: $10.1051 /$ epjconf/201610605006

(C) Owned by the authors, published by EDP Sciences, 2016

\title{
Improvement of Nuclear Heating Evaluation Inside the Core of the OSIRIS Material Testing Reactor
}

\author{
Arthur Péron ${ }^{\mathrm{a}}$, Fadhel Malouch, and Cheikh M. Diop \\ Alternative Energies and Atomic Energy Commission (CEA), Saclay center, \\ DEN/DANS/DM2S/SERMA, 91191 Gif-sur-Yvette Cedex, France
}

\begin{abstract}
In this paper we present a nuclear heating from neutron and photon rays calculation scheme mainly based on the Monte-Carlo neutral particle transport code TRIPOLI- $4{ }^{\circledR}$ which takes into account the axial distributions of fuel element compositions. This calculation scheme is applied to the OSIRIS reactor in order to evaluate the effect of using realistic axially heterogeneous compositions instead of uniform ones. After a description of nuclear heating evaluation, the calculation scheme is described. Numerical simulations and related results are detailed and analysed to determine the impact of axially heterogeneous compositions on fluxes, power and nuclear heating.
\end{abstract}

\section{Introduction}

Technological neutron/photon irradiations undertaken in experimental reactors are essential for the safety for the current power reactor fleet as well as for testing innovative types of reactors. Irradiation programs carried out in Material Testing Reactors (MTR) must be representative of phenomena and irradiation conditions in actual power plants, in particular in terms of neutron flux (or fluence), pressure and temperature. Material samples irradiated inside a MTR core needs to have their irradiation conditions enslaved to fixed values. Temperature closed-loop control is obtained by electric heating elements or by modifying the nature of a gas surrounding the sample. This corresponds for about $20 \%$ of the total temperature in irradiation samples. The other $80 \%$ comes from the nuclear heating due to the energy deposited by neutrons and photons in samples. Nuclear heating varies approximately from 2 to $16 \mathrm{~W} / \mathrm{g}$ and depends mainly on the sample nature and location in the reactor core. A reliable evaluation of such heating is also one of the key data for thermal studies in the design and safety of experimental devices.

The purpose of this paper is to highlight the effects of using an axial profile of compositions instead of a uniform composition for fuel elements in nuclear heating calculations using the Monte-Carlo neutron and photon transport code TRIPOLI-4 [1]. In this paper, the nuclear heating evaluation is first described and then the nuclear heating calculation scheme is detailed. Afterwards, the OSIRIS MTR and its modeling for the TRIPOLI-4 code are presented. Then the effect of composition axial profiles on fluxes, power and nuclear heating are investigated and analysed. Finally, a comparison of results versus measurements is made.

\footnotetext{
${ }^{a}$ Corresponding author: ARTHUR. PERON@cea.fr
}

This is an Open Access article distributed under the terms of the Creative Commons Attribution License 2.0, which permits unrestricted use, distribution, and reproduction in any medium, provided the original work is properly cited. 


\section{Nuclear Heating Evaluation Using the TRIPOLI- $4^{\circledR}$ Code}

\subsection{Deposited Energy Calculation}

We recall here the basic definitions and properties of deposited energy KERMAs induced by a neutron flux in a coupled neutron-photon transport [2]. Nuclear heating due to neutrons can be conveniently expressed in terms of the KERMA factors $k_{i, j}(\mathrm{E})$, i.e., Kinetic Energy Release in Matter where $E$ is the incident particle energy. The definition of the kermas $k_{i, j}(E)$ is such that the heating rate $H(E)$ of a mixture of different materials in a given volume reads at incident energy $E$ :

$$
H(E)=\sum_{i} \sum_{j} \rho_{i} k_{i, j}(E) \varphi(E) .
$$

Here $\rho_{i}$ is the atom density of material $i, \varphi(E)$ is the neutron (scalar) flux at $E$ and $k_{i, j}(E)$ is the kerma factor for element $i$ and reaction $j$ at $E$. Thus, the kermas play the role of a microscopic reaction cross section, carrying units of [energy $\times$ cross section], i.e., $[\mathrm{MeV} \times \mathrm{b}]$. In other words, the kerma factor $k_{i, j}(E)$ expresses the total kinetic energy release $\bar{E}_{i, j}(E)$ induced by the reaction $j$ in the element $i$, at a given incident energy $E$, times the microscopic cross section $\sigma_{i, j}(E)$ for the same nuclear reaction in the same material:

$$
k_{i, j}(E)=\sum_{c} \bar{E}_{i, j}^{c}(E) \sigma_{i, j}(E)
$$

where the sum is performed over all charged products resulting from the nuclear reaction $j$, including the recoil nucleus. By definition, the term $\bar{E}_{i, j}^{c}(E)$ expresses the kinetic energy carried away by the $c^{\text {th }}$ charged reaction product. The cross sections act as weight factors, in that they express the probability that each of the possible reactions contributes to the total energy release.

For most isotopes or natural elements in nuclear data libraries, however, detailed information for $\bar{E}_{i, j}^{n}(E)$ is not available, so that the kerma factors must actually be computed via the energy balance method. This amounts to noting that the energy allocated to charged reaction products and recoil nuclei is obtained by subtracting the energy carried away by transported neutrons and photons from the available initial (mass and kinetic) energy. It is crucial to recall that both neutrons and photons are assumed to be transported. The available energy for the reaction is given by the sum $+Q_{i, j}$, where $Q_{i, j}$ is the reaction Q-value resulting from the mass defect (in energy units), measured with respect to the ground state of each nucleus. We have then:

$$
E+Q_{i, j}=\sum_{c} \bar{E}_{i, j}^{c}(E)+\bar{E}_{i, j}^{n}(E)+\bar{E}_{i, j}^{\gamma}(E),
$$

so that we obtain the neutron KERMAs:

$$
k_{i, j}(E)=\left[E+Q_{i, j}-\bar{E}_{i, j}^{n}(E)-\bar{E}_{i, j} \gamma(E)\right] \sigma_{i, j}(E)
$$

where $\bar{E}_{i, j}^{n}$ is the mean energy carried away by the emitted neutrons (if any) and $\bar{E}_{i, j}^{\gamma}(E)$ is the mean energy carried away by the emitted photons (if any). In both $\bar{E}_{i, j}^{n}(E)$ and $\bar{E}_{i, j}^{\gamma}(E)$ terms, multiplicity is also taken into account. Equation (4) provides the energy balance method that is currently used in TRIPOLI-4 to compute the energy deposition [2].

According to the ENDF format [3], for all reactions $j$ on the element $i$, the required cross sections $\sigma_{i, j}(E)$ can be retrieved from nuclear data libraries in section MF3, and are associated to a distinct MT number. Each MT number identifies a reaction with specific incoming particle, reaction products and residual nucleus (depending on the target nucleus $i$ ). The $\mathrm{Q}$-value $Q_{i, j}$ is coded in the header of each MT reaction in MF3, but TRIPOLI-4 computes $Q_{i, j}$ by resorting to mass defect calculations whenever possible. 


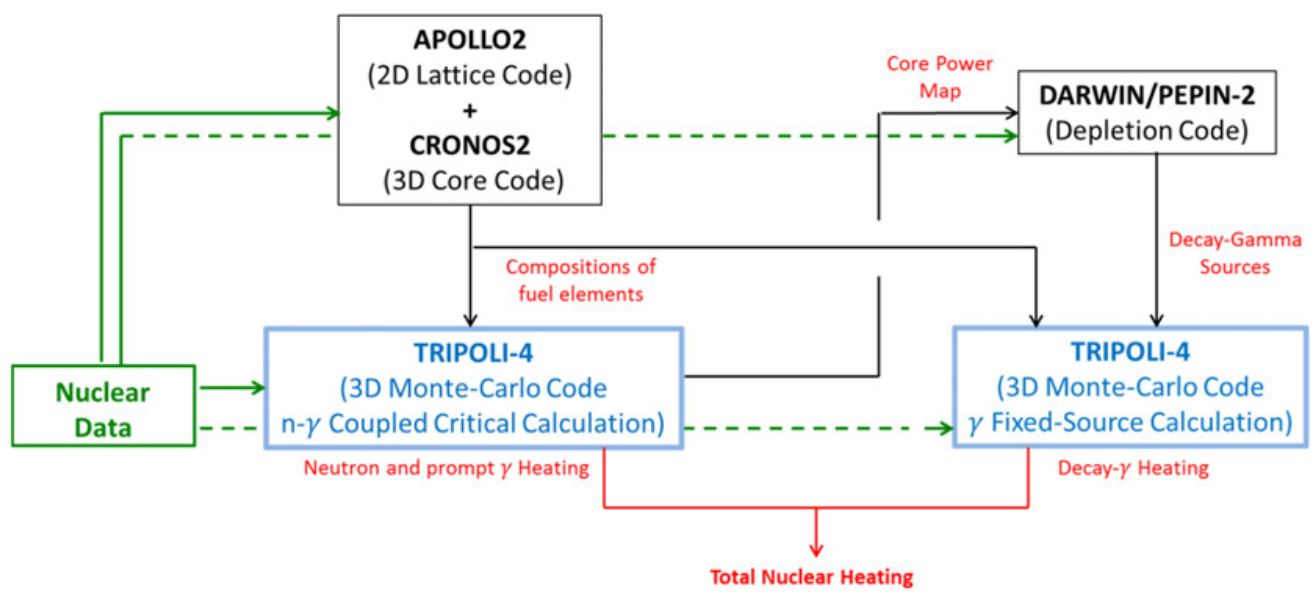

Figure 1. Nuclear heating evaluation scheme architecture.

Energy deposition calculation is strongly correlated to nuclear data evaluations. A number of isotopes of the most common data libraries, such as for instance JEFF-3.1.1, JEFF-3.2 and ENDF/ B-VII.1 [4], are affected by energy and/or momentum conservation faults in the photon productions, which potentially result in negative kermas (typically when the photon energy exceeds the limit allowed by kinematics), sharp glitches (missing photons demanded by energy conservation), or inaccurate thresholds for photon emission sections $[5,6]$. Such inconsistencies can be detected for instance by resorting to the kinematic limit method [7].

\subsection{Coupled Neutron-photon 3D Calculation Scheme}

To evaluate nuclear heating in reactors, a three-dimensional coupled neutron-photon calculation scheme has been set up. The calculation scheme architecture is presented in Fig. 1. It is based mainly on the TRIPOLI-4 Monte Carlo transport code which has been extensively validated against experimental results from neutron flux measurements performed in ex-core and in-core experiments [8, 9]. Two TRIPOLI-4 simulations are needed to estimate the total nuclear heating. The first one is a coupled neutron-photon critical calculation for the nuclear heating due to neutrons and prompt photons. The second one is a photon fixed source calculation which simulates gamma from fission products decays. These two main steps need incoming data: nuclear data which represent the physics of simulations and irradiation data for setting up geometry and compositions of the core. The calculation scheme uses nuclear data from the CEAV5_1.1 library, based mainly on the nuclear data library JEFF3.1.1 [10]. Irradiation data are either given by the user (control elements positions, loading of experimental devices... ) or computed by some deterministic calculation codes.

Until now, evaluations of compositions were done by a 2D core code coupled with the APOLLO2 lattice code thus nuclear heating calculations were necessarily done with a uniform composition along the $\mathrm{Z}$ axis [11]. The purpose of this paper is to highlight the interest of realistic 3D irradiation conditions to estimate particle fluxes, power and nuclear heating for neutrons, prompt photons and decay photons. Estimations of fuel elements compositions are now performed with the APOLLO2 2D lattice code and the CRONOS2 3D core code. The APOLLO2 code calculates multi-group self-shielded cross sections on a fine energy grid (281 groups) for the different elements in the core. Cross sections are then condensed and stored in libraries called SAPHYBs to be used by the CRONOS2 code. CRONOS2 allows the following of fuel elements evolution along their subsequent irradiations by chained calculations. 


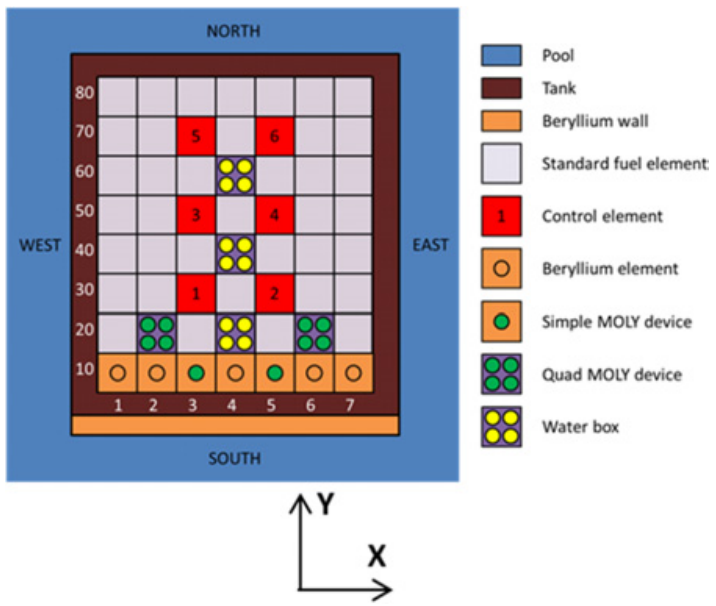

Figure 2. Radial cross-section of the OSIRIS core (a number is associated at each position given by the sum of the column and the line numbers. For example here we have 3 water boxes in positions 24, 44 and 64).

It permits to estimate axial distributions of burn-up and compositions for each fuel element at a chosen time. This is a major innovation for nuclear heating evaluation in the case of the OSIRIS reactor.

\section{Relevance of Axial Distributions of Compositions for Fuel Element, Application on the OSIRIS MTR Reactor}

\subsection{TRIPOLI-4 Modeling of the OSIRIS Reactor}

The OSIRIS Material Testing Reactor (MTR) is an experimental pool type light water reactor operational since 1966 at the CEA Saclay center. The core is a compact unit of $60 \mathrm{~cm} \times 70 \mathrm{~cm} \times 70 \mathrm{~cm}$ for a power of $70 \mathrm{MW}$. The core vessel contains a rack of 56 cells loaded with 38 standard fuel elements made of 22 fuel plates, 6 control elements (hafnium absorber above a fuel element) and up to 7 beryllium elements (Fig. 2). Two cells (22 and 26) are employed for the radioisotope production with medical use (MOLY devices). Cells 24, 44 and 64 are loaded with water boxes dedicated to experiments (up to four experiments rigs by water box).

The modeling of the OSIRIS core is as close to the plans as possible (Fig. 3), for example each fuel plate is taken into account, control elements can be moved or axial discretization of fuel element compositions can be modified.

This part will show comparisons between three modeling: a calculation with an axially uniform composition for fuel elements and two calculations with a fuel composition axial discretization of 7 and 21 cells. Comparisons are done for all core elements on an axial grid of 21 meshes containing the effective geometry. In the following we present results for two fuel elements and one irradiation element (water box) representative of phenomena. Fuel elements are in cells 34 and 84 (Fig. 2) with respectively a mean burn-up of 80 and $15 \mathrm{GW}$.day/ton and the water box is in cell 44 . In this study we have evaluated the following quantities:

- neutron fluxes, prompt and decay photon fluxes, powers (obtained with fission reaction rates), neutron nuclear heating and prompt and decay photon nuclear heating for each fuel element on an axial mesh of 21 cells

- fluxes and nuclear heating for neutron, prompt photon and decay photon in the water box. In the study, Monte Carlo standard deviations are for all calculated tallies less than $1 \%$. 


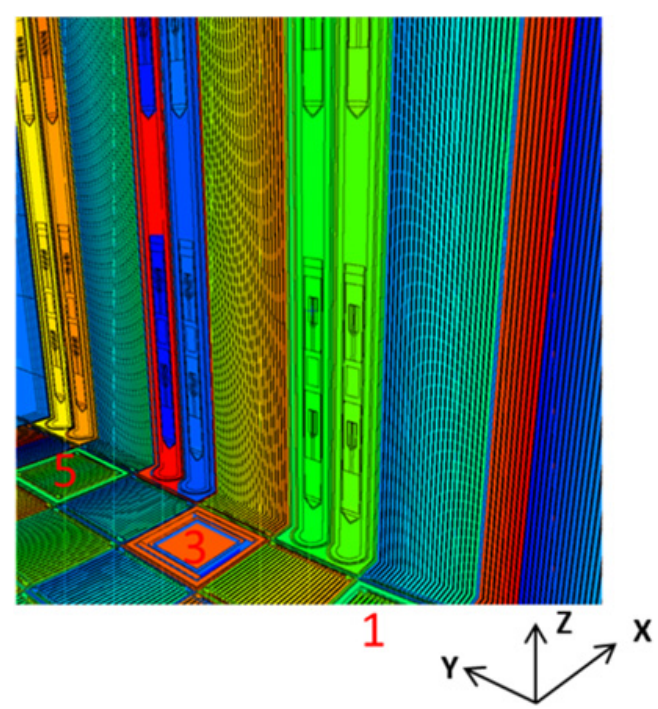

Figure 3. 3D modeling of the OSIRIS core for TRIPOLI-4 (red number refer to control element positions from Fig. 2),

\section{Fuel Element in cell $34(80 \mathrm{GWj} / \mathrm{t})$}

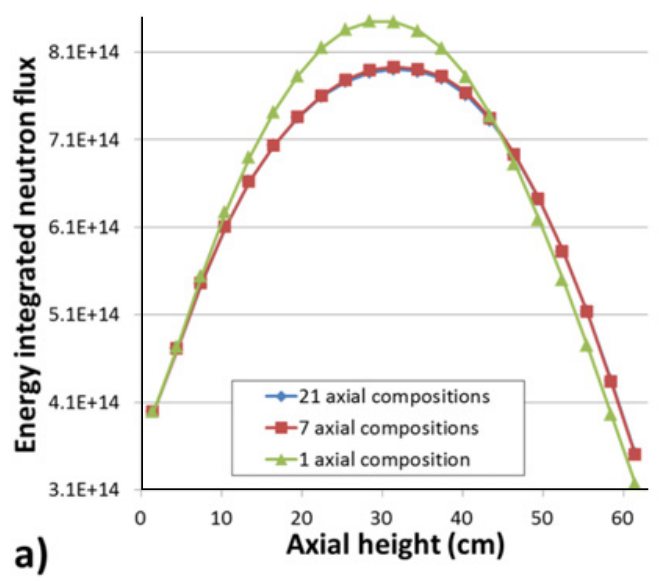

Fuel Element in cell $84(15 \mathrm{GWj} / \mathrm{t})$

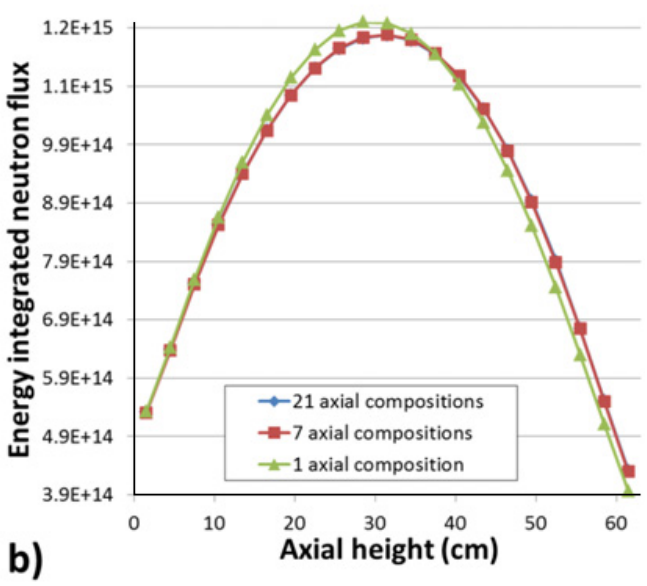

Figure 4. Neutron axial distribution flux (integrated from 0 to $20 \mathrm{MeV}$ ) by axial mesh for 2 fuel elements at different burn-up (a) $80 \mathrm{GW} . \mathrm{d} / \mathrm{t}$, (b) $15 \mathrm{GW} . \mathrm{d} / \mathrm{t}$.

\subsection{Neutron and Photon Fluxes Comparisons in Fuel Elements}

Figure 4 shows that using axial distributions instead of an axially uniform composition altered the neutron flux axial profile. This phenomenon mainly depends on fuel element burn-up and increases with it. It is explained by an increase of neutron poison production and a loss of fissile elements in the middle of fuel elements during their life due to a more important neutron flux at this location. So, for a high burn-up fuel element, neutron poisons are in higher concentrations at the centre while fissile elements are in reduced quantities. We can notice that 7 and 21 compositions cells gives similar results in term of neutron flux profiles and this is similar for prompt photons and decay photons. 

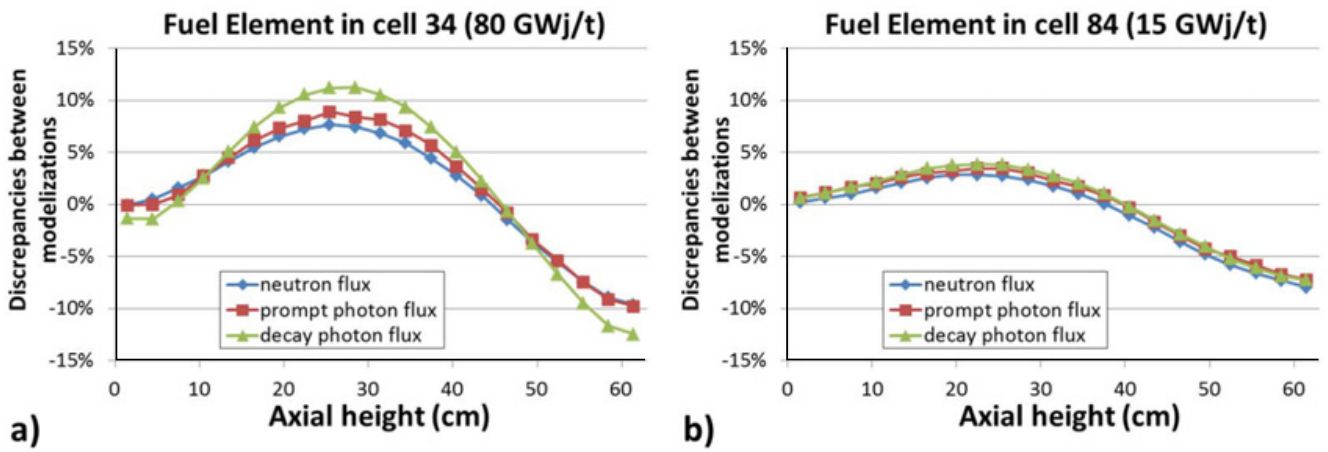

Figure 5. Flux discrepancies between 1 versus 21 cells modeling for 2 fuel elements at different burn-up (a) $80 \mathrm{GW} . \mathrm{d} / \mathrm{t}$, (b) $15 \mathrm{GW} . \mathrm{d} / \mathrm{t}$.
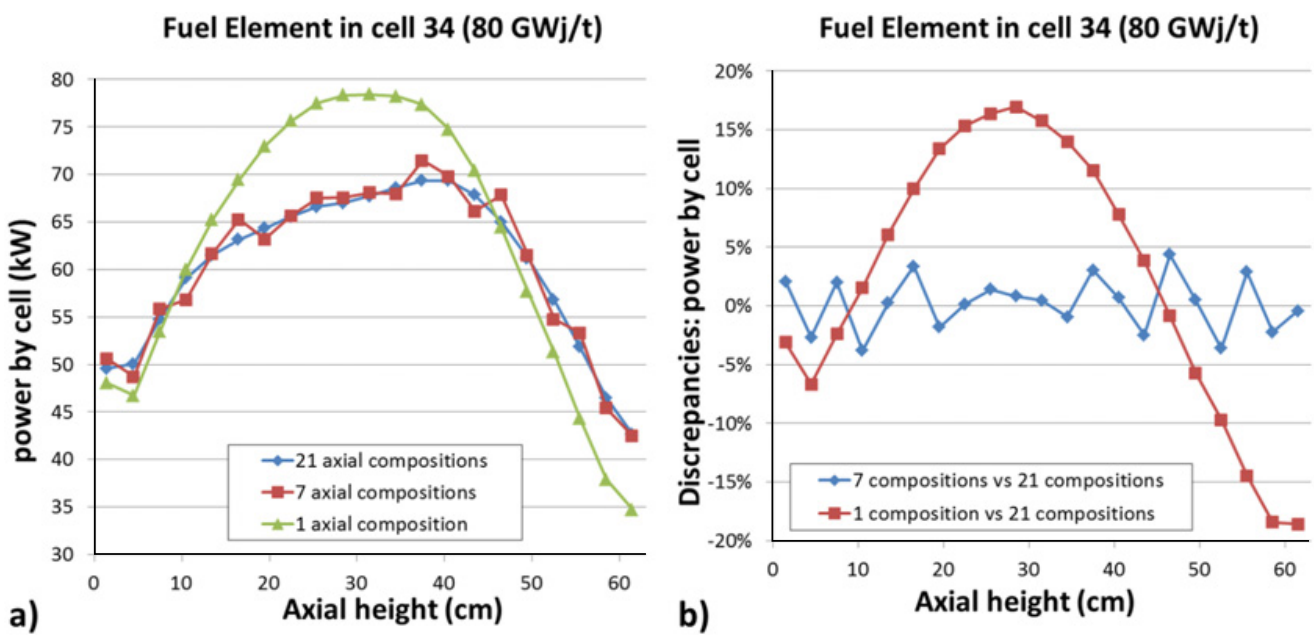

Figure 6. a) Power by cell for the 3 different modeling b) discrepancies between power of the different modeling.

Figure 5 quantifies flux discrepancies between uniform axial compositions versus axial profile of 21 compositions. It highlights that fuel elements with high burn-up have their different fluxes (neutron, prompt photon and decay photon) affected by the axial distribution of compositions, but not in same term. Decay photon flux is the more affected (discrepancies from $-12 \%$ to $12 \%$ ) due to the important changes in term of fission products concentrations. Discrepancies on neutron and photon fluxes are similar (from $-10 \%$ to $8 \%$ ) because prompt photons are mainly created by neutron reactions.

\subsection{Power and Deposited Energy Comparisons in Fuel Elements}

In fuel elements, powers and deposited energies are more affected than fluxes by a change in the axial distribution of compositions. First, there are more discrepancies between 7 and 21 axial compositions in term of power as shown in Fig. 6. Secondly, with an axial distribution of concentrations, the axial power profile shape is more degraded and loses his symmetry. It corresponds more at expected results for the core geometry. Indeed, control elements in the upper part of the core reduce fissile elements loss by fission while under irradiation. Thus, upper part of fuel elements at high burn-up gives more power 


\section{$15^{\text {th }}$ ISRD}

Fuel Element in cell $34(80 \mathrm{GWj} / \mathrm{t})$

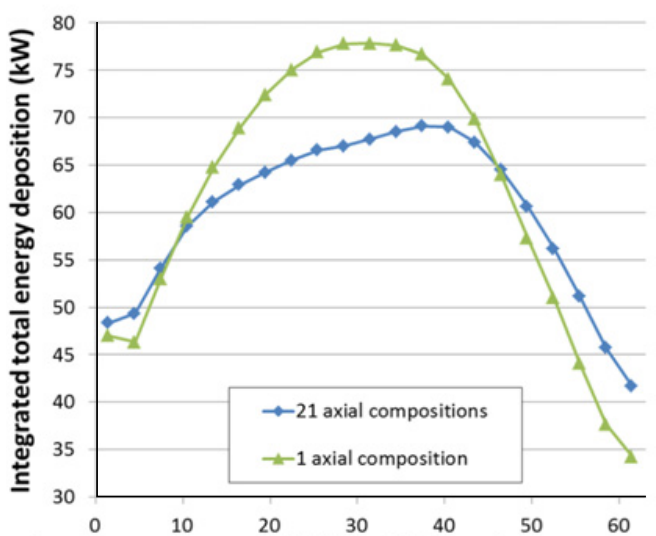

a)

Axial height $(\mathrm{cm})$

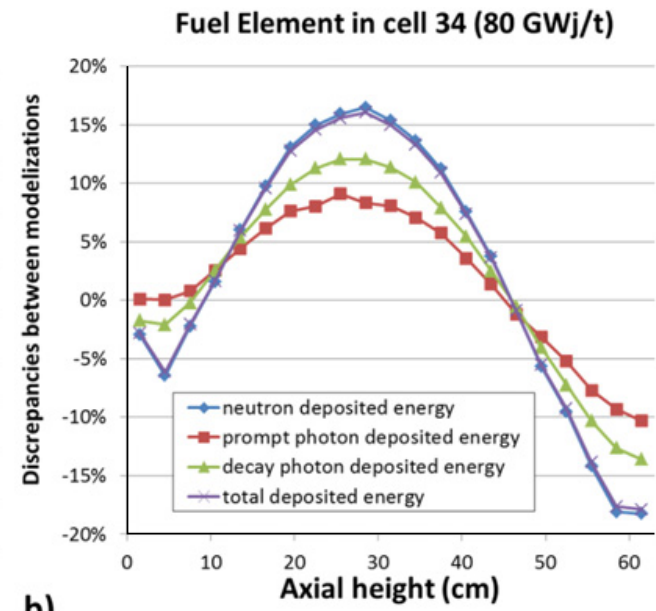

Figure 7. a) Total nuclear heating axial profile for 2 modeling b) discrepancies for the different nuclear heating contributions (1_cell/21_cells - 1).
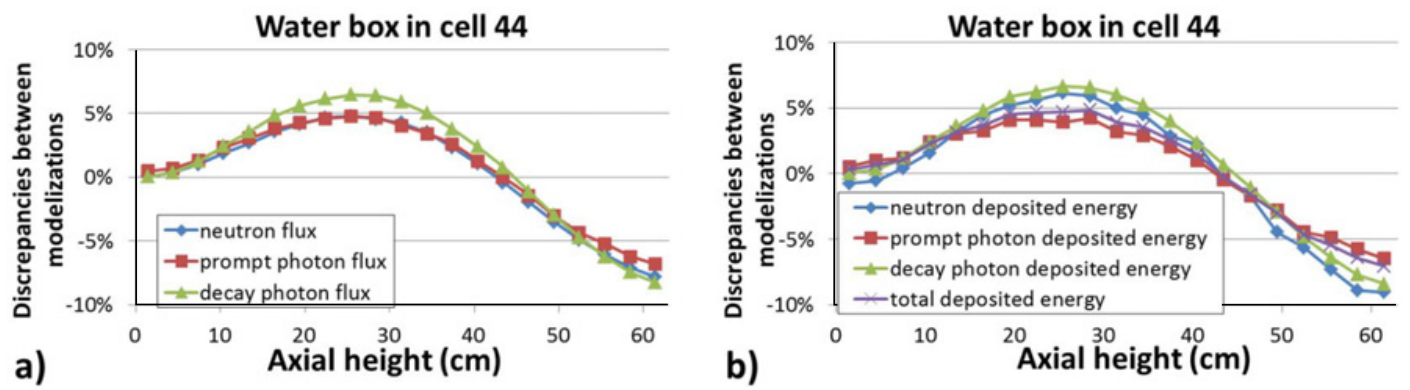

Figure 8. Discrepancies between 1 axial composition versus 21 compositions in water box in cell 44 a) fluxes b) deposited energies.

than lower part. Discrepancies between 1 versus 21 compositions modeling are in range of $-18 \%$ to $17 \%$.

Nuclear heating and power are strongly correlated in fuel elements. Indeed, the major part of deposited energy comes from fission products which carry about $170 \mathrm{MeV}$ from the $200 \mathrm{MeV}$ Q-value. In that, power is close to neutron nuclear heating which represent about $95 \%$ of total nuclear heating. Thus, total nuclear heating axial profile (Fig. 7a) in fuel elements is similar as the profile power. Concerning photon nuclear heating, prompt photons discrepancies they vary from $-10 \%$ to $9 \%$ while decay photons discrepancies are in range of $-14 \%$ to $12 \%$.

\subsection{Fluxes and Deposited Energy Comparisons in Water Box}

Results in experimental positions are less affected by axial compositions than fuel elements. Discrepancies are in range of $-10 \%$ to $7 \%$ as shown in Fig. 8. They correspond to the core average discrepancies. These results are comparisons between calculations and need to be confronted to experiment. This has been done in the next part. 


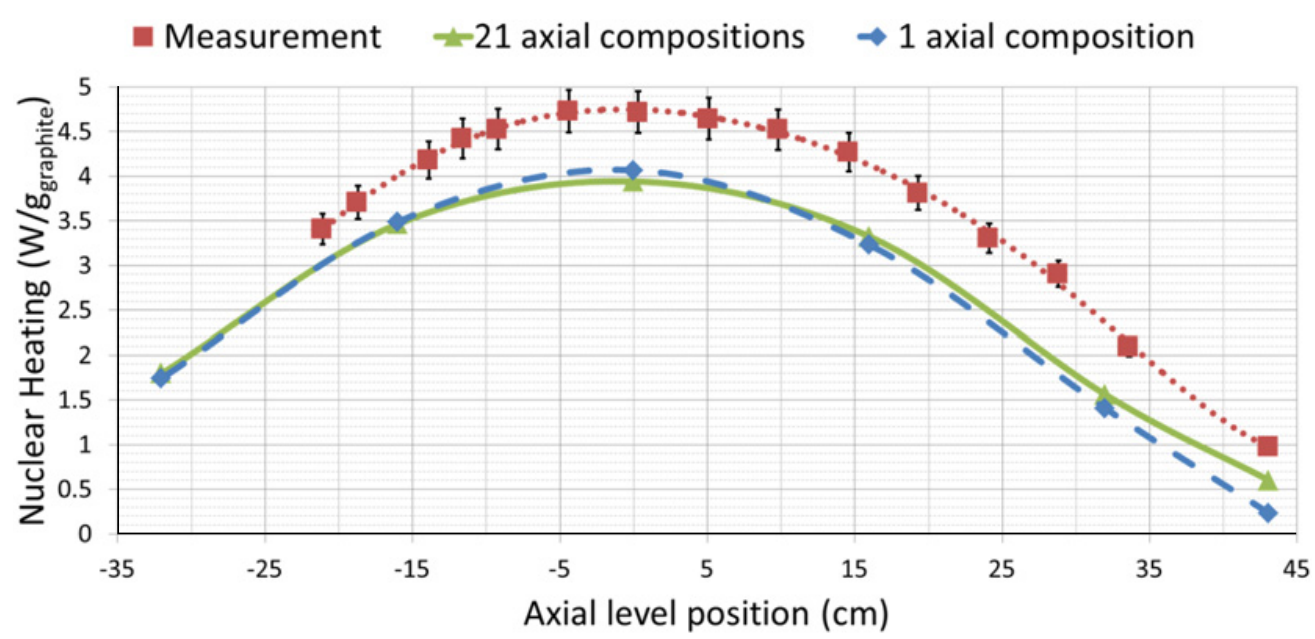

Figure 9. Comparison between experimental measurements and calculation results in position 44SO of the OSIRIS core.

\section{Experimental Validation Using Differential Calorimetry}

To complete this study we have set up a comparison of the three modeling versus differential calorimetry results performed with the CALMOS experimental device [12, 13]. The principle of differential calorimetry is to compare the temperatures of a cell filled with a sample (of a given material) to a reference cell (with no sample). The temperature difference is directly correlated to the nuclear heating in the sample. In OSIRIS calorimeter devices, nuclear heating is measured in graphite samples because of its low atomic number which disturb not much the photon flux and its good transparency to neutron. Figure 9 shows results of measurements versus calculation results. We can see that using an axial profile for compositions changes the shape of nuclear heating in calorimeter, significantly reducing discrepancies with measurements for well above reactor mid-plane (from-76\% to $-39 \%$ at $43 \mathrm{~cm}$ ). However, discrepancy at core mid-plane is lightly increase from $-14 \%$ to $-16.5 \%$.

\section{Conclusions and Prospects}

In this paper we present a nuclear heating calculation scheme which takes into account fuel elements axial distributions of compositions. We apply the calculation scheme to the OSIRIS reactor and evaluate the effect of using realistic axially heterogeneous compositions instead uniform ones by calculation/calculation comparisons. Impacts are evaluated in fuel and experimental elements on fluxes, power or nuclear heating. This permits to highlight the importance of realistic compositions to take into account the good axial profile of calculated quantities which can be misevaluated by $\pm 20 \%$. However, this is a local phenomenon which mainly depends on fuel element burn-ups and poison productions and whole core is affected in lower proportion $(-12 \%$ to $7 \%)$.

An experimental validation has been set up to complete this study. Results are compared to nuclear heating measurements performed using differential calorimetry in an experimental location. Discrepancies between measurements and calculation results are in range of $-15 \%$ at core mid-plane and they are strongly reduced with the use of realistic compositions for the upper part of the core (from $-76 \%$ to $-39 \%$ ). 


\section{$15^{\text {th }}$ ISRD}

Sources of discrepancies between experiments and simulations could be explained by a lack in photon production nuclear data [7] and simplifications concerning modeling. Further investigations are ongoing to understand these discrepancies.

\section{References}

[1] TRIPOLI-4 Monte Carlo Transport Code, http://www.nea.fr/abs/html/nea-1716.html (2013)

[2] A. Zoia et al., Energy deposition in TRIPOLI-4, version 8, Rapport CEA 11-5165 A (2011)

[3] National Nuclear Data Center, Brookhaven National Laboratory. ENDF-6 Formats Manual. Data Formats and Procedures for the Evaluated Nuclear Data File ENDF/B-VI and ENDF/B-VII. CSEWG Document ENDF-102, Report BNL-90365-2009

[4] M.B. Chadwick et al., ENDF/B-VII.1 Nuclear Data for Science and Technology: Cross Sections, Covariances, Fission Product Yields and Decay Data, LA-UR 11-05121, LANL, USA, 2011

[5] http://t2.lanl.gov/nis/data/endf/ebalVII.1/summary.html

[6] N. J. Peters, J. C. McKibben, K. Kutikkad, and W. H. Miller, Refining the Accuracy of Predicting Physics Parameters at Research Reactors due to the Limitations in the Energy Balance Method Using MCNP and the ENDF Evaluations, Nuclear Science And Engineering: 171, 210-219 (2012)

[7] A. Péron, F. Malouch, A. Zoia, C. M. Diop, "Impact Estimation of Nuclear Data Inconsistencies on Energy Deposition Calculations in Coupled Neutron-Photon Monte-Carlo Simulation, with TRIPOLI-4®", Joint International Conference on Supercomputing in Nuclear Applications and Monte Carlo 2013 (SNA+MC 2013), Paris, France, October 27-31, 2013

[8] H. Carcreff, A. Alberman, L. Barbot, F. Rozenblum, D. Beretz and Y. K. Lee, "Dosimetry Requirements for Pressure Vessel Steels Toughness Curve in the Ductile to Brittle Range", J. of ASTM International (JAI), Vol. 3, Issue 3, (Mar., 2006)

[9] Y.K. Lee and F. Malouch, "Analysis of OSIRIS In-Core Surveillance Dosimetry for GONDOLE Steel Irradiation Pragram by Using TRIPOLI-4 Monte Carlo Code", 13th International Symposium on Reactor Dosimetry (ISRD13), Akersloot, Netherlands, May 21-25, 2008

[10] Santamarina A., Bernard D., Blaise P., Coste M., Courcelle A., Huynh T.D., Jouanne C., Leconte P., Litaize O., Mengelle S., Noguère G., Ruggieri J.M., Sérot O., Tommasi J., Vaglio-Gaudard C., Vidal J-F., "The JEFF-3.1.1 Nuclear Data Library, JEFF Report 22, Validation Results from JEF-2.2 to JEFF-3.1.1," NEA No. 6807, OECD/NEA Edition 2009

[11] F. Malouch, "Development and Experimental Validation of a Calculation Scheme for Nuclear Heating Evaluation in the Core of the OSIRIS Material Testing Reactor", Journal of ASTM International (JAI), Volume 9, Issue 4 (April 2012)

[12] H. Carcreff, "CALMOS: Innovative Device for the Measurement of Nuclear Heating in Material Testing Reactors", in Journal of ASTM International (JAI), Volume 9, Issue 3 (March 2012)

[13] H.Carcreff, L.Salmon, C. Courtaux, "First In-Core Measurement Results Obtained with the Innovative Mobile Calorimeter CALMOS inside Osiris Material Testing Reactor", Advancements in Nuclear Instrumentation Measurement Methods and their Applications (ANIMMA-2013), 23-27 June 2013, Marseille 